\title{
Distant cancer effects on standardised testing of peripheral vision
}

William W Dawson, Berry L Jordan, Robert D Marsh, Kaushik Hazariwala, Franklin P Flowers, Ting Fang

\begin{abstract}
Background-Profound central-retinal visual losses have been a major presenting factor reported in cancer and melanoma associated retinopathies (CAR, MAR). However, it is well established that standardised tests of peripheral retinal function are often the most sensitive detectors of early eye disease. This is a preliminary investigation of the responsiveness of the peripheral retina to "distant" (non-eye or CNS) cancers using easily obtained standardised tests.

Methods-The design is a single blind study where test results are compared with published norms and a small age matched control group. Of 120 ambulatory cancer outpatients who were interviewed at routine follow up examinations, 111 volunteered and admitted a range of mild visual changes. 25 cancer patients completed all tests of peripheral vision function and a clinical screening. There were seven control subjects of the same age range.
\end{abstract}

Results-98\% (49 of 50) of eyes from the patient cohort were judged clinically normal following examinations which emphasised the central retina, fundus appearance, and static fields. On testing which emphasised the visual periphery, 46 $(92 \%)$ eyes showed one or more quantitative abnormalities $>2$ SD from the age adjusted norm means. These abnormalities clustered mainly about dark adaptation (rod cell) sensitivity (31, 62\% of measured sites), the blue sensitive retinal cells $(17,34 \%$ of measured eyes), and the oscillatory component (OP) of the electroretinogram $(23,46 \%$ of measured eyes). One control eye ( $7 \%$ ) showed a significant dark adaptation abnormality and ERG reduction. There was no identifiable interaction between chemotherapy mode and the cancer associated retinal deficits (CARD). Antiretinal antibodies were found in sera from most patients and controls.

Conclusion-CARD is common in the retinal periphery of many cancer patients, and is distinct from rare CAR, MAR central-retinal responses. CARD has numerous potential clinical uses which justify expanded research with more defined large samples.

(Br f Ophthalmol 2001;85:291-296)
It has been several years since the first report of rare but serious visual losses which occur as paraneoplastic retinopathies ${ }^{1}$ associated with carcinoma of the lung ${ }^{2}$ and less often with cervix, colon, prostate, and breast cancers. ${ }^{3}$ Cancer associated retinopathy (CAR) is characterised by rapid onset, progressive acuity loss, and mild to moderate fundus changes ${ }^{1}$ without invasion of the eye by cancer ("distant cancer"). A related and even more rare retinopathy has been associated with malignant melanoma of the skin (MAR). ${ }^{5}$ MAR is typified by the sudden onset of night "blindness", bizarre visual images, and "moving" lights. Circulating factors and autoimmune responses at various retinal sites have been implicated in both diseases. ${ }^{78}$

Patients seldom seek specialty eye care before there is a significant change in central vision, as in CAR or MAR. However, in some patients with retinal disease, subtle abnormality may often be detected first in the periphery. There are a number of well established measures of peripheral function that are widely appreciated for low variability, quantitative results, and large published norms. We have used several of these quantitative tests to explore the pathophysiology of early onset cancer associated peripheral retinal deficits.

\section{Methods and subjects}

Bias possibly associated with specialty referrals was avoided by seeking patients at the Shands Cancer Center outpatient clinics and who, on questioning, reported changes in "vision" associated with onset of their cancer. Subjects at this tertiary care, academic centre were from 120 subjects seen sequentially in the outpatient cancer clinics; 111 gave informed consent, described minor visual disturbances (itching, floaters, fatigue, reduced night sensitivity, and occasional blurriness), and completed an interview about their general medical status and visual condition. No patient had clinically detectable haematological disease or metastatic disease to the nervous system or the eye as judged by the oncology service and later by the eye service. Of the 111 subjects, 25 aged 27-69 years completed clinical eye evaluations, clinical testing, peripheral vision testing, and immunological evaluation. Scheduling problems and transportation were the main limiters of the cohort size. Seven volunteers, aged 37-59 years, without cancer history and claiming normal vision were enrolled as controls. All procedures were approved by the Shands Hospital institutional review board.

Routine clinical tests were supervised by the retina service chief. Patients and controls had 
Table 1 Distribution of antiretinal antibodies by cancer diagnosis

\begin{tabular}{|c|c|c|c|c|c|c|c|c|c|c|c|c|c|c|}
\hline \multirow[b]{2}{*}{$\begin{array}{l}N \\
(k D a)\end{array}$} & \multicolumn{2}{|c|}{ Control } & \multicolumn{2}{|c|}{ Breast } & \multicolumn{2}{|c|}{ Melanoma } & \multicolumn{2}{|c|}{ Lung } & \multirow{2}{*}{$\begin{array}{l}\text { Testicular } \\
1 \\
\text { IGM IGG }\end{array}$} & \multirow{2}{*}{$\begin{array}{l}\text { Bladder } \\
1 \\
I G M I G G\end{array}$} & \multicolumn{2}{|c|}{ Renal } & $\begin{array}{l}\text { Ewing's } \\
\text { sarcoma }\end{array}$ & Ovarian \\
\hline & $\begin{array}{l}7^{\star} \\
I G M\end{array}$ & $I G G$ & $\begin{array}{l}10 \\
I G N\end{array}$ & $I G G$ & $\begin{array}{l}7 \dagger \\
I G M\end{array}$ & $I G G$ & $\begin{array}{l}3 \\
I G M\end{array}$ & $I G G$ & & & $\begin{array}{l}1 \\
I G N\end{array}$ & $I G G$ & $\begin{array}{l}1 \\
I G M I G G\end{array}$ & $\begin{array}{l}1 \\
I G M I G G\end{array}$ \\
\hline $10-$ & & & & & & & & & & & & & & \\
\hline $20-$ & & & 20 & & 20 & 22 & & & 20 & & & & 22 & \\
\hline & 24 & & & 24 & & 24 & & & & & & & & \\
\hline & 25 & & 25 & & 25 & & & & & & & & & \\
\hline & 26 & & 26 & 26 & & & 26 & 26 & & & 26 & & & 26 \\
\hline & 27 & & & & & & & & & & & & & 27 \\
\hline & 28 & & 28 & 28 & 28 & & 28 & & & 28 & & & & 28 \\
\hline $30-$ & 30 & 30 & 30 & 30 & 30 & 30 & 30 & $\begin{array}{l}30 \\
34\end{array}$ & 30 & 30 & 30 & 30 & 30 & 30 \\
\hline $40-$ & & 40 & & 38 & & & & & & & 38 & & & \\
\hline & 44 & 44 & & $\begin{array}{l}42 \\
44\end{array}$ & & 44 & & & & & & & & \\
\hline & 46 & 46 & & 46 & 46 & 46 & 46 & 46 & & 46 & 46 & 46 & 46 & 46 \\
\hline & 48 & 48 & & & & & & & & & & & & \\
\hline $\begin{array}{l}50- \\
60-\end{array}$ & & & & & & & & & & & & & & \\
\hline $70-$ & $\begin{array}{l}67 \\
70\end{array}$ & & & 67 & & & & & & & & & & \\
\hline $80-$ & 80 & & 80 & 80 & 80 & & 80 & & 80 & & & & & \\
\hline
\end{tabular}

${ }^{\star}$ Negative result.

†Negative results.

All serum dilutions were $1 / 400$.

complete clinical examinations of both eyes including visual acuity, slit lamp examination, refraction, ocular motility, dilated fundus examination, colour vision screening (Ishihara pseudoisochromatic plates), and static visual perimetry (Humphrey full threshold program 24-2 or Goldmann fields). ${ }^{9}$ Serum samples were drawn to evaluate the possibility of immune system involvement. Finally, each eye was clinically rated as "normal" or "abnormal".

Periphery testing ${ }^{9}$ included a quantitative measure of colour discrimination FarnsworthMunsell 100 hue test (FM) with computer scoring, Goldmann-Weekers type dark adaptation thresholds, and retinal electrical signal production, electroretinography (ERG). Technical personnel had no information about the patients' clinical conditions.

ERGs were stimulated according to international standards (IS $)^{10}$ with retinal signals recorded from the corneal apex referenced to the outer canthus. The photopic full field light adaptation produced a retinal illumination of approximately 700 trolands. Dark adapted ERG responses were recorded after 15 minutes of darkness. Photopic ERG signals were stimulated by white xenon flashes presented at $1 / 4 \mathrm{~Hz}$. Intensity ranged in $0.3 \mathrm{log}$ unit steps to three $\log$ units below the IS stimulus $\left(2 \mathrm{~cd} / \mathrm{m}^{2} / \mathrm{s}\right){ }^{10}$ The eyes were also stimulated at $30 \mathrm{~Hz}$ at the IS intensity. Photopic oscillatory potentials (OPs) were recorded by rejecting signal components below $70 \mathrm{~Hz}$ elicited by the IS stimulus at $1 / 4 \mathrm{~Hz}$. Macular responses $\left(4^{\circ}\right)$ were stimulated by light flashes at $32 \mathrm{~Hz}$ at IS. Dark adapted signals were recorded at IS and at 0.5 $\log$ unit steps to $3.5 \mathrm{log}$ units below the IS intensity.

The curve of visual threshold change during dark adaptation was drawn for each eye using the Goldmann-Weekers adaptometer (HaagStreit, Berne, Switzerland). ${ }^{9}$ After 5 minutes of light adaptation at $670 \mathrm{~cd} / \mathrm{m}^{2}$, the threshold decrease in darkness was measured at two $11^{\circ}$ diameter retinal sites $\left(12^{\circ}\right.$ superior, $40^{\circ}$ temporal) over a period of 30 minutes. Extensive norms also exist for dark adaptation. The Haag-Streit, Goldmann-style adaptometer has produced several sets of standards which show standard deviation (SD) limits of approximately $0.2 \log$ units about the mean at the asymptote of sensitivity gain after 27 minutes of dark adaptation. ${ }^{11}{ }^{12}$ A large colour norm database is well established for the FM test. ${ }^{13} 14$ The FM provides a scaled quantification of colour discrimination along the primary colour axes. It has the advantages of reliability, a Gaussian distribution of normal results, and general acceptance. Graphics, statistical evaluations, and norm based decisions were automated. ${ }^{14}$ The ERG norms used in this research were generated from the IS prescribed stimuli within the University of Florida Eye Center electrodiagnostic laboratory and consists of mean and variance distributions for 33 eyes age of 9-59. They are consistent with an independent norm group of 538 eyes. ${ }^{15}$ Results from the periphery tests will be presented only if they are more than 2 SD from their published norm means.

\section{Results}

No control subjects showed clinical or bilateral peripheral retinal changes of function. One eye (7\%) showed a scotopic ERG reduction and reduced dark adapted sensitivity. Three (12\%) in the cancer patient cohort had received no chemotherapy. Six (24\%) had received no form of chemotherapy in more than 12 months. All others $(64 \%)$ had received one or more of 13 agents for chemotherapy more than 21 days before testing. Clinical results show that two ( $4 \%$ ) of 50 eyes had best corrected visual acuities of $\leqslant 20 / 40$ (Snellen notation). Of these, one was determined to be amblyopic and one had a cataract. Three $(6 \%)$ of 50 had $20 / 30$ acuity with the remainder $(90 \%)$ having visual 

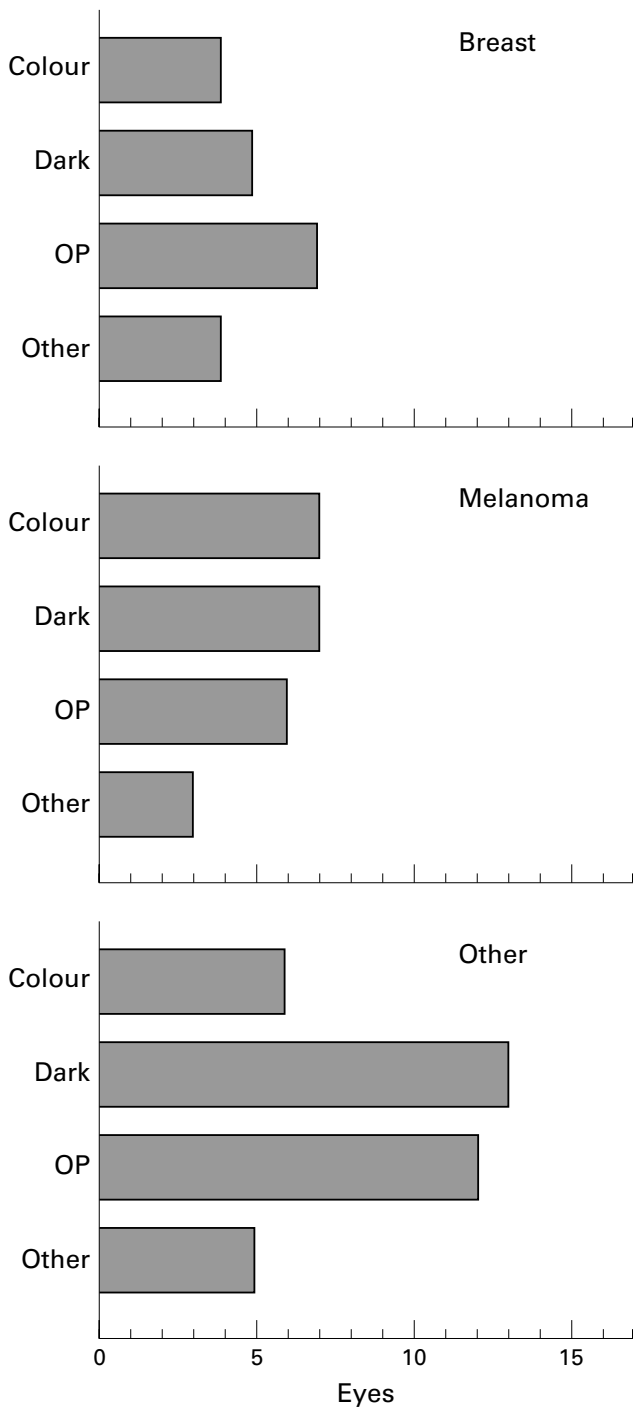

Figure 1 Visual abnormalities in cancer patients. Distribution of patient eyes by diagnosis and visual deficits $>2$ SD below the norm means. Colour confusion (Colour), electroretinogram high pass signal (OP), final dark adapted sensitivity (dark), deficit bar widths indicate the number of eyes representing a specific diagnostic category. Minor, less numerous deficits are lumped as "other". A variety of small sample cancers are represented under "other". These include lung, testicular, cervical, bladder, renal, and Ewing's sarcoma.

acuity of $20 / 25$ or better. Nine eyes (18\%) had $20 / 15$ acuity. We found one slightly constricted field (orbital oedema following superficial surgery) and a fundus with macular drusen. All colour vision responses to the Ishihara pseudoisochromatic plates were normal. Finally, $49 / 50(98 \%)$ eyes (one amblyope) of the 25 volunteer cohort were reported (double blind) as clinically normal by the eye service.

Antinuclear antibody (ANA) titre was negative in $19(76 \%)$ cancer subjects or had a titre of $1 / 40$ in six (24\%) subjects. Samples were also examined for immune responses against western blots of normal donor human retina proteins. Antiretina antibodies were found in all but two $(92 \%)$ subjects (Table 1), but no correlation was found between the autoimmune profile and visual test findings. There were no responses against the CAR protein (23 $\mathrm{kDa}$ recoverin). ${ }^{16}{ }^{17}$ Thirteen different antiretina antibodies were found in $6 / 7$ of the control samples.

Seven of the 50 eyes but none of the 25 patients tested completely within $<2 \mathrm{SD}$ of norm limits. However, all the scotopic ERG signals (time and amplitude) were normal. Three melanoma patients had one eye with normal results while the other eye showed either a tritan colour defect or significantly elevated final dark adaptation threshold. All other patients showed either defects in both eyes or multiple monocular abnormalities. The most common abnormalities were the tritan colour defect, elevated final dark adaptation threshold, and reduced OP amplitudes. Figure 1 summarises the distribution of major deficits arranged by cancer diagnosis.

Table 2 shows the number of significant $(2$ SD) positive eye findings. Colour test results were analysed and assigned to a colour confusion class by computer comparison with published, age corrected norms. ${ }^{18-20}$ We found no protan or deutan errors. Only tritan axis and non-axial errors indicating general hue confusion were detected. The OP, macular (Mac), and $30 \mathrm{~Hz}$ findings all relate to the ERG signal components.

Figure 2 shows typical FM colour sequence error scores. Colour space is represented by a numerical system which progresses anticlockwise around the circumference of the colour space circles in Figures 2A-C. Figure 2B shows a normal result with random errors produced by non-systematic mistakes. This figure is from a patient with breast cancer who, not withstanding the age of 62 years, has a normal low error score of 44. Larger deviations from random errors occur in Figure 2A. Here, common sex linked protan and deutan errors from two (non-study) eyes are superimposed. Figure 2C shows a set of tritan errors distributed along the blue axis of the FM colour space between positions 34 and 49, but note errors elsewhere in colour space. This patient had a history of testicular cancer and an acuity in this eye of $20 / 20$. This error score is significantly different from the norm group $(p=0.05)$ after correction for age.

Figure 3 is a chart produced by the Goldmann-Weekers adaptometer. Dark adaptation as light (sensitivity gain) threshold reduction proceeds through a predictable sequence of changes after the adapting field is turned off ( 5 minutes). The broad curve shown in Figure 3 describes the normal mean range, threshold decline plus or minus 2 SD. ${ }^{14}$ Local

Table 2 Cancer types and associated visual deficits

\begin{tabular}{lrrccll}
\hline Diagnosis & Patients & DA & Colour & OP & Mac & $30 \mathrm{~Hz}$ \\
\hline Melanoma & 7 & 7 & 7 & 6 & 1 & 2 \\
Breast & 10 & 5 & 4 & 7 & 4 & 0 \\
Lung & 3 & 5 & 2 & 3 & 0 & 0 \\
Other & 5 & 8 & 4 & 7 & 4 & 1 \\
Totals & 25 & 25 & 17 & 23 & 9 & 3
\end{tabular}

All deficit results are $>2$ SD from the norm mean. Patients had one or more defective eyes.

$\mathrm{DA}=$ final dark adapted threshold elevation, number of eyes. Colour $=$ tritan or non-axial defect, number of eyes. $\mathrm{Mac}=$ macular local $\left(4^{\circ}\right)$ ERG potential reduction, number of eyes. 30 $\mathrm{Hz}=$ reduced ERG responses to $30 \mathrm{~Hz}$ flashing stimuli, number of eyes. 
A

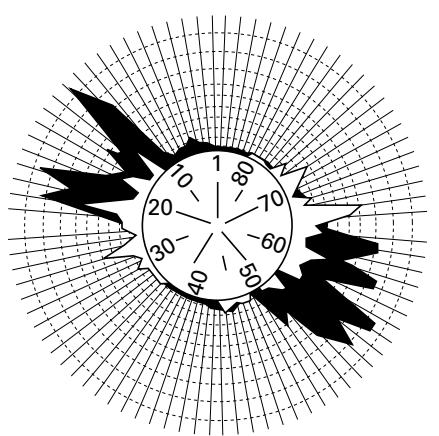

B

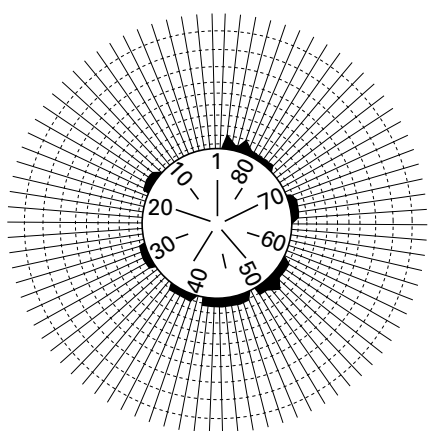

C

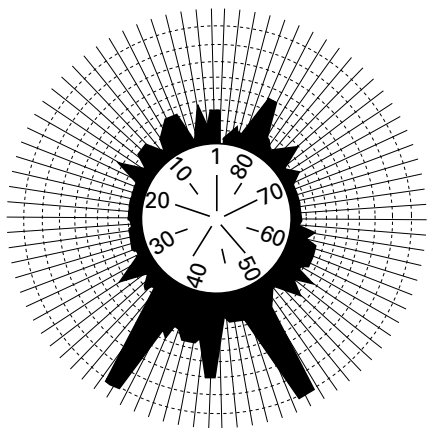

Figure 2 Common colour sensitivity deficits. Typical distributions of colour confusion in the colour space configured by the FM test and its norm database. (A) Common male protan (white) together with deutan error axes (black). Protan is the distribution with the peak at 67\%. (B) Non-axial scatter produced a normal error score (breast cancer patient). (C) Tritanopic defect (testicular cancer patient) with peaks in the blue axis region of colour space loci 34-49. Other errors in (C) show general colour confusion.

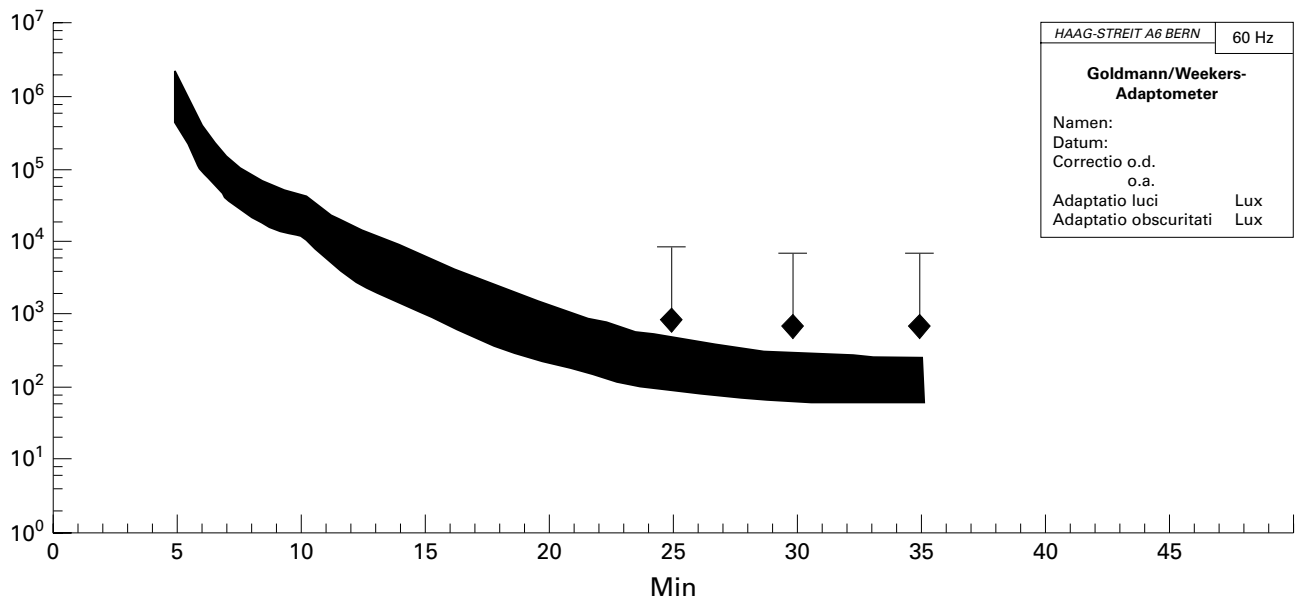

Figure 3 Dark adaptation in cancer patients. Goldmann-Weekers dark adaptation threshold record. Darkness began at 5 minutes after 1000 trolands full field light adaptation. The ordinate is log threshold for light detection. Abcissa is time. The dark band is the means plus or minus 2 SD for two norm groups, one generated internally and one external. ${ }^{11}$ Points (diamond) at 25, 30, and 35 minutes represent thresholds from the cancer patient sample presented as mean and plus 2 $S D$.

laboratory norms fit comfortably on top of the published data. Age less than 75 years is not a significant factor in normal dark adaptation. ${ }^{14}$ Normal eyes produce similar dark adaptation results at the $15^{\circ}$ superior and $40^{\circ}$ temporal test sites; we have used both as "independent" data points from each eye. Then the threshold values (log units) of available patient eye data points represented by Figure 3 means are at 25 minutes, $\mathrm{n}=98$, mean $=2.89, \mathrm{SD}=0.59 ; 30$ minutes, $\mathrm{n}=98$, mean $=2.74, \mathrm{SD}=0.58 ; 35$ minutes, $\mathrm{n}=76$, mean $=2.71, \mathrm{SD}=0.51$. At 25 minutes, $61 / 98$ data points from the cancer patients are more than 2 SD above the norm mean (grey zone centre). Similarly, 51/98 and 41/76 are above norm limits after 30 and 35 minutes in darkness.

Measures of the dark adapted ERG signals were not discriminating. The scotopic ERG b-wave has been reported to be the most age dependent ERG component in normals. However, the mean amplitude loss is not significant until after age 55 and the variability is large. ${ }^{15}$ Yet, the dark adapted b-wave does provide for an objective validation of the relation between retinal tissue function (ERG) and the DA subjective threshold data (Fig 3). To make this comparison DA thresholds were sorted by

magnitude. The upper 12 and lower 12 threshold data sets were separated and the ERG b-waves results collected. Following an ANOVA (repeated measures design), a Tukey protected $t$ test $(t=2.29, \mathrm{p}<0.05)$ showed that the $b$-waves were larger from the eyes with greater subjective gain in DA sensitivity.

From Table 2, the ERG component which discriminated best was the photopic OP response, where 23 (46\%) eyes showed significant abnormality. The major deficits cluster (DA, Colour, OP) in the periphery. DA test sites were peripheral, short wavelength (tritan) weakness originates from s-cones which are most dense outside the fovea and OPs have not been produced in humans by local fovea stimulation.

\section{Discussion}

The paraneoplastic eye diseases melanoma associated retinopathy (MAR) and cancer associated retinopathy (CAR) typically show rapid onset, pronounced visual effects and fundus changes in the clinic. ${ }^{1-8}$ Both have been associated with immune responses. Table 1 shows that a number of circulating antiretinal antibodies are present in most $(23,92 \%)$ but 
not all of the cancer patients in this study. Most frequent weights are at 30,46 , and $80 \mathrm{kDa}$. Some samples show no clear distribution pattern. We were surprised to find antiretinal antibodies in $6 / 7$ of the visually normal controls. However, it has been known for some time $^{21}$ that naturally occurring antibodies may be found directed against a great variety of antigens (often self antigens) in normal human sera in the absence of apparent functional consequences. A recent editorial ${ }^{22}$ reported that 15 cancer associated retinal antigens have been identified. However, specific linking to functional changes is incomplete. Others report antiretinal antibodies in controls. A brief survey of recent papers on cancer associated retinal antigen/antibody research shows that the question of responsiveness to "normal" sera is handled in many ways. Eleven papers did not examine responses to normal sera. One used three normal controls but did not describe the results. ${ }^{23}$ One used controls with diagnosed diabetic or aged related retinopathy and reported (except at $23 \mathrm{kDa}$ ) no differences between cancer patients and controls. ${ }^{24}$ While others have reported multiple retinal antigen/ antibody reactions from their normal controls. ${ }^{725}$ The reason for these reactions to normal sera is not clear as is the absence of reaction in some individuals. Also in contrast with CAR and MAR general ophthalmic clinical evaluations did not detect any differences between our cohort of cancer volunteers and normals for colour discrimination, general eye health, static fields, Snellen acuity, and dilated fundus examination. After more than a year from periphery testing clinical records were examined post mortem in 10 of our study patients. None reported late complaints typical of CAR or MAR.

Peripheral function measurements detected one or more early peripheral visual deficits in most of the cancer patients. Two (20\%) breast cancer patients with the most marginal deficits were near the edge of the second SD for OPs and Mac ERG potentials in a single eye. Most other patients $(22 / 25)$ had at least two robust peripheral deficiencies and $7 / 25$ had three or more. These are large numbers which encourage a search for errors.

Age and chemotherapy treatment toxicity could easily be sources of bias. However, age is factored into all norm group comparisons. Further, several eyes with pronounced tritan defects were among the younger in the group. All were from melanoma patients aged 27-39. The patient aged 27 showed marked bilateral tritan defects. A 36 year old patient with melanoma who had only surgical treatment and no chemotherapy showed large OP abnormalities and DA threshold losses in both eyes. Of the other young melanoma patients, two had received interferon treatments. One had a unilateral tritan defect. Varied unilateral defects were found in six patients. Two relatively young patients ( 51 and 55 years) with bladder cancer and renal cancer had been treated with very different chemotherapeutic regimens but showed the same deficit cluster (tritan, OP,
DA). Other patients with histories of only surgical or no treatment showed both losses of DA sensitivity and changes in ERG signal components in the absence of tritanopia.

A less likely bias source is the presence of congenital deficits caused by sampling error. A very strong example is provided by the remarkable findings of tritanopia in clinically "normal" eyes. The probability of finding a congenital tritan defect is approximately $1 / 20000 .{ }^{26}{ }^{27}$ The cumulative probability associated with finding 17 tritans in our unrelated cohort becomes vanishingly small. In contrast, no common congenital protan or deutan defects were encountered in our sample. Monocular deficits seem to rule out congenital contamination. Some patients had one eye (7, $14 \%$ ) which showed no peripheral abnormality. Three were melanoma patients. Two of these showed unilateral tritan confusion and another showed a very large (1 log unit) differential loss of final dark adapted sensitivity. The remaining four showed mixed losses of monocular OP components, macular $4^{\circ}$ signal, and tritan defects.

The most common clusters of two or three visual deficits include reduced DA sensitivity and a colour confusion, all easily relatable to receptor cells. However, eight eyes with no other signs showed significant reductions in photopic oscillatory potentials of the electroretinogram. This implicates deep retina pathology. Basic electrophysiology studies using intraretinal micropipettes report that the OPs originate from neurons of the inner retina, ganglion cell inner plexiform layer region. ${ }^{28}$ The amplitudes of the OPs have been reported to be highly sensitive and diagnostic of congenital stationary night blindness and mild retinal ischaemia. ${ }^{29}$ It is reported that OPs can be used to predict the onset of proliferative retinopathy in insulin dependent diabetic patients months before there are other clinical signs. $^{30} 31$

The chemotherapy modes for this group of patients were mixed, ranging from no treatment to complex treatment. Involved at one time was cisplatin, interferon, methotrexate, vincristine, carboplatin, doxetaxel, paclitaxel, cis-retinoic acid, cyclophosphamide, doxorubicin, topotecan, vinorelbine, and tamoxifen. A number of these were used sequentially or simultaneously. Four of these compounds have a history of rare retinal toxicity, but only in high doses. Ripps et $a b^{\beta 2}$ reported a condition similar to recessively inherited night blindness in a patient following vincristine chemotherapy for malignant melanoma. Ten cases of retinal ischaemia were reported by one group ${ }^{33}$ when interferon was used to treat cancer and several other diseases. The most common finding was retinal "cotton wool spots", together with local haemorrhages and/or vascular congestion. The only function measures were visual acuity which ranged from $20 / 20$ to $20 / 400$. One report of retinal toxicity has been published which describes a case of ovarian cancer and an inadvertent overdose of cisplatin. ${ }^{34}$ Her ERG b-waves were of the (abnormal) negative type as described in vincristine intoxication. Early acuity was 20/400, 
bilaterally. Colour vision was defective with a tritan axis. After some months her acuity returned to normal but the ERG remained abnormal together with the tritan defect.

For retinal toxicity, the most studied anticancer agent is probably tamoxifen. One review $^{35}$ concludes that ocular toxicity is very rare with clinical evaluations such as we have described as part of this study. ${ }^{36}$ Another study ${ }^{37}$ of 303 women added a few visual function tests. All patient groups (in cancer care) showed a median colour confusion score greater than 18, which is abnormal for women in this age group when the test is scored as described by Bowman. ${ }^{38}$ In our cohort the single patient with a tamoxifen treatment history was colour normal. We found no chemotherapeutic agents which produce a toxic response fingerprint consistent with the deficit clusters that we have described. Curiously, this cluster of peripheral findings is closely duplicated by findings from patients with very early insulin dependent diabetic retinopathy. ${ }^{14} 30$ Several centres have reported similar findings over a number of years. ${ }^{3032} 39$

Unlike the scenario that pertains in rare "cancer associated retinopathy" or "melanoma associated retinopathy" 6 traditional clinical indicators of eye disease, acuity, fundus appearance, intraocular pressure, static perimetry, and pseudoisochromatic plates do not detect the pericentral cancer associated retinal deficits (CARD) that we have reported. Measures of components of peripheral retinal function are more productive. In time, large cohort studies of cancers at prediagnosis, treatment, and post-treatment stages are needed to clearly define the clinical promise of our findings. Many more data are needed before the efficiency and validity of application to specific cancers can be reported to the clinical community. Now, our sample predicts that CARD deficit clusters are especially likely in patients with breast cancer and cutaneous melanoma. For them the loss of tritan and scotopic sensitivity may have immediate counselling value for occupational or driving safety.

Our data raise questions about common factors and call for new attention to the participation of the retina and other central nervous system tissues in cancer associated neoplastic syndromes. We hope this path will lead to new methods for the detection and measurement of subclinical cancer.

This research was supported in part by a grant to the department of ophthalmology from Research to Prevent Blindness and the Dr Martin and Edith L Horowitz memorial fund. None of the authors has commercial or proprietary interest in any of the drugs mentioned in this study, in the company that produces them, or in any competing company.

We are indebted to R Taher, MD, C Weeks, MD, T Maida, L Shaver, and R Carrol, MD, for assistance in testing and patient Shaver, and R Carrol, MD, for assistance in testing and patient
records/management. We also appreciate the effort by B DeSue records/management. We also appreciate the effort

and T Barnash in production of the manuscript.
Presented at the 1998 meeting of Association for Research in Vision and Ophthalmology at Ft Lauderdale, Florida, USA.

1 Sawyer RA, Selhorst JB, Zimmerman LE, et al. Blindness caused by photoreceptor degeneration as a remote effect of caused by photoreceptor degeneration as
cancer. Am 7 Ophthalmol 1984;8:606-13.

2 Buchanan TAS, Gardiner TA, Archer DB. An ultrasound study of retinal photoreceptor degeneration associated with bronchial carcinoma. Am f Ophthalmol 1984;97:277-87.
3 Thirkill CE, Keltner JL, Tyler NK, et al. Antibody reactions with retina and cancer-associated antigens in 10 patients with cancer-associated retinopathy. Arch Ophthalmol 1993; 111:931-7.

4 Thirkill CE, Rother AM, Keltner JL. Cancer associated retinopathy. Arch Ophthalmol 1987;105:372-5.

5 Ripps H, Carr RE, Siegel IM, et al. Functional abnormalities in vincristine-induced night blindness. Invest Ophthalmol Vis Sci 1984;25:787-94.

6 Berson EI, Lessell S. Paraneoplastic night blindness with malignant melanoma. Am f Ophthalmol 1988;106:307-11.

7 Milan AH, Saan JC, Jacobson SG, et al. Antibodies against retinal bipolar cells in cutaneous melanoma-associated retinal bipolar cells in cutaneous melanoma-associa
retinopathy. Invest Ophthalmol Vis Sci 1993;34:91-100.

8 Thirkill CE, Fitzgerald P, Sergott RC, et al. CancerThirkill CE, Fitzgerald P, Sergott RC, et al. Cancer-
associated retinopathy (CAR syndrome) with antibodies reacting with retinal, optic nerve, cancer cells. $N$ Engl f Med 1989;321:1589-94.

9 Weleber RG and Eisner A. Retinal function and physiological studies. In: Newsome DA, ed. Retinal dystrophies and degenerations. New York: Raven Press, 1988:21-69.

10 Marmor MF, Zrenner E. Standard for clinical electroretinography (1994 update). Doc Ophthalmol 1995;89:199-210.

11 Herse P. A new method for quantification of the dynamics of dark adaptation. Optom Vis Sci 1995;72:907-10.

12 Möntyjärvi $M$. Colour vision and dark adaptation in diabetic patients after photocoagulation. Acta Ophthalmol 1989;67:113-8.

13 Verriest G, Van Laethem J, Uvijls A. A new assessment of the normal ranges of the Farnsworth-Munsell 100 hue test. Am F Ophthalmol 1982;93:635-42.

14 Benzschaivel T. Computerized analysis of the FarnsworthMunsell 100 hue test. Am $\mathcal{F}$ Optom Physiol Optics 1985;62: 254-64.

15 Birch DG, Anderson JL. Standardized full-field electroretinography. Arch Ophthalmol 1992;110:1571-6.

16 Matsubara S, Yamaji Y, Sato M, et al. Expression of a photoreceptor protein, recovering, as a cancer-associated retinopathy autoantigen in human lung cancer lines. Br f Cancer opathy autoantigen

17 Adamus G, Ortega H, Witkowska D, et al. Recoverin: A potent uveitogen for induction of photoreceptor degeneration in Lavis rats. Exp Eye Res 1994;59:447-56.

18 Farnsworth D. The Farnsworth-Munsell 100 hue and dichotomous tests for color vision. F Opt Soc Am 1942;33: $568-78$

19 Kinnear PR. Proposals for scoring and assessing the 100-hue test. Vis Res 1970;10:423-33.

20 Aspinall PA. Inter-eye comparison on the 100 hue test. Acta Ophthalmol 1974,52:307-13.

21 Guilbert B, Dighiero G, Avrameas S. Naturally occurring antibodies against nine common antigens in human sera. $\mathscr{f}$ Immunol 1982;128:2779-87.

22 Keltner J, Thirkill C. Cancer-associated retinopathy vs recoverin-associated retinopathy. Amf Ophthalmol 1998; 126:296-302.

23 Rizzo J, Gittinger J. Selective histochemical staining in the paraneoplastic retinopathy syndrome. Ophthalmology 1992; 99:1286-93.

24 Thirkill C, Kettner J, Tyler N, et al. Antibody reactions with retina and cancer-associated antigens in 10 patients with cancer-associated retinopathy. Arch Ophthalmol 1993:111: 931-7.

25 Adamus G, Aptsiauri N, Guy J, et al. The occurrence of serum autoantibodies against enolase in cancer-associated retinopathy. Clin Immunol Immunopathol 1996;78:120-9.

26 Wright WD. The characteristics of tritanopia. f Opt Soc Am $1952 ; 42 \cdot 509-21$.

27 Kalmus $\mathrm{H}$. The familial distribution of congenital tritanopia. Ann Hum Gen 1955;20:39-56.

28 Kozak W. Electroretinogram and spike activity in mammalian retina. Vis Res 1971;(Suppl) 3:129-49.

29 Simonsen SE. Prognostic value of ERG oscillatory potential in juvenile diabetics. Acta Ophthalmol 1980;58:865-78.

30 Bresnick G, Korth K, Groo A, et al. Electroretinographic oscillatory potentials predict progression of diabetic 1307-11.

31 Bresnick GH, Palta $\mathrm{H}$. Predicting progression of severe proliferative diabetic retinopathy. Arch Ophthalmol 1987;105: $810-4$.

32 Ripps H, Carr RE, Siegel IM, et al. Functional abnormalities in vincristine-induced night blindness. Invest Ophthalmol Vis Sci 1984:25:787-94.

33 Guyer DR, Tiedeman MD, Yannuzzi LA et al. Interferonassociated retinopathy. Arch Ophthalmol 1993;111:350-6.

34 Marmor MF. Negative-type electroretinogram from cisplatin toxicity. Doc Ophthalmol 1993;84:237-46.

35 Nayfield SG, Gorin MB. Tamoxifen-associated eye disease: a review. F Clin Oncol 1996;14:1018-26.

36 Tang R, Shields J, Schiffman J et al. Retinal changes associated with tamoxifen treatment for breast cancer. Eye 1997; 11:295-7

37 Gorin M, Day R, Constantino J, et al. Long term tamoxifen citrate use and potential ocular toxicity. Am $\mathcal{f}$ Ophthalmol 1998;125:493-501.

38 Bowman KJ. A method for quantitative scoring of the Farnsworth Panel D-15. Acta Ophthalmol 1982;60:907-16.

39 Hardy KJ, Lipton J, Scase MO, et al. Detection of color vision abnormalities in uncomplicated type 1 diabetic patients with angiographically normal retinas. $\mathrm{Br} \quad \mathcal{F}$ Ophthalmol 1992;8:461-4. 